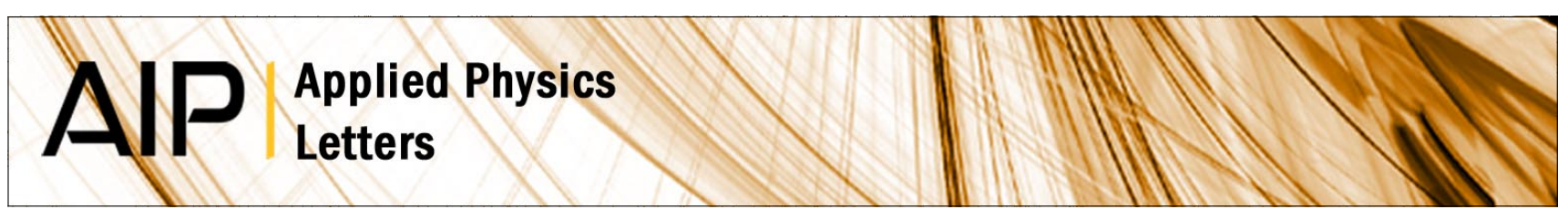

\title{
Buckling-driven delamination of carbon nanotube forests
}

Parisa Pour Shahid Saeed Abadi, Shelby B. Hutchens, Julia R. Greer, Baratunde A. Cola, and Samuel Graham

Citation: Appl. Phys. Lett. 102, 223103 (2013); doi: 10.1063/1.4802080

View online: http://dx.doi.org/10.1063/1.4802080

View Table of Contents: http://apl.aip.org/resource/1/APPLAB/v102/i22

Published by the AIP Publishing LLC.

Additional information on Appl. Phys. Lett.

Journal Homepage: http://apl.aip.org/

Journal Information: http://apl.aip.org/about/about_the_journal

Top downloads: http://apl.aip.org/features/most_downloaded

Information for Authors: http://apl.aip.org/authors

\section{ADVERTISEMENT}



\title{
Buckling-driven delamination of carbon nanotube forests
}

\author{
Parisa Pour Shahid Saeed Abadi, ${ }^{1}$ Shelby B. Hutchens, ${ }^{2}$ Julia R. Greer, ${ }^{2}$ \\ Baratunde A. Cola, ${ }^{1,3, a)}$ and Samuel Graham ${ }^{1,3, a)}$ \\ ${ }^{1}$ George W. Woodruff School of Mechanical Engineering, Georgia Institute of Technology, 771 Ferst Drive, \\ Atlanta, Georgia 30332, USA \\ ${ }^{2}$ California Institute of Technology, 1200 E. California Blvd. MC 309-81, Pasadena, California 91125, USA \\ ${ }^{3}$ School of Materials Science and Engineering, Georgia Institute of Technology, 771 Ferst Drive, Atlanta, \\ Georgia 30332, USA
}

(Received 13 February 2013; accepted 1 April 2013; published online 4 June 2013)

\begin{abstract}
We report buckling-driven delamination of carbon nanotube (CNT) forests from their growth substrates when subjected to compression. Macroscale compression experiments reveal local delamination at the CNT forest-substrate interface. Results of microscale flat punch indentations indicate that enhanced CNT interlocking at the top surface of the forest accomplished by application of a metal coating causes delamination of the forest from the growth substrate, a phenomenon not observed in indentation of as-grown CNT forests. We postulate that the post-buckling tensile stresses that develop at the base of the CNT forests serve as the driving force for delamination. (C) 2013 AIP Publishing LLC. [http://dx.doi.org/10.1063/1.4802080]
\end{abstract}

Carbon nanotube (CNT) forests are composed of nominally vertically aligned, ultra-high aspect ratio nanotubes with various degrees of alignment and entanglement along their height. ${ }^{1-6}$ Their unique thermal, electrical, photonic, and mechanical properties, which in part arise from the complex nano- and micro-structural detail of these materials, render them potential candidates for many applications. Their mechanical response, which potentially affects the functionality of CNTs in these applications, has been investigated by various methods including nano- and micro-scale indentation of films of CNT forest and compression of pillars comprised of CNT forest. ${ }^{1,4-31}$ The deformation mechanism was found to be dominated by periodic buckling in the case of CNT forest micro-pillars ${ }^{5,11,12,14,31}$ and by the combination of buckling and shear offsets in flat punch indentations of CNT forest films. ${ }^{1,4,10,13,17,19}$ While the visualization of nanoscale and microscale compression of CNT forests adds useful insight into the mechanics of these structures, it is not able to capture all deformation. Alternate failure modes may occur during the deformation of larger scale CNT forests that could be relevant to component or system level applications.

We report on the delamination of CNT forest films as revealed by macro-compression experiments. Postcompression scanning electron microscope (SEM) images of $1 \mathrm{~cm} \times 1 \mathrm{~cm}$ CNT forests reveal local delamination of the films at nominal pressures as low as $50 \mathrm{kPa}$. Delamination driven by in-plane buckling has been observed in monolithic thin films where the biaxial compressive residual stress in the film led to local buckling. This buckling subsequently drove lateral propagation of the delamination at the interface. ${ }^{32,33}$ Buckling in the case of monolithic films is the "thin plate" type of buckling, i.e., in the direction perpendicular to the film surface. In contrast to this mechanism, the delamination of CNT forests reported here, is related to the column buckling of high aspect ratio CNTs in the direction

\footnotetext{
${ }^{\text {a) }}$ Authors to whom correspondence should be addressed. Electronic addresses: sgraham@gatech.edu or cola@gatech.edu.
}

perpendicular to the CNTs, i.e., parallel to the film surface. We show that the occurrence of delamination is facilitated by the bending moments acting on the CNT-substrate interface as a result of local buckling. We also show that stress concentrations associated with the non-uniformity of CNT heights play a role in delamination during macrocompression. Furthermore, we demonstrate a method for tailoring the degree of interaction among CNTs to cause delamination in micro-scale indentation of CNT forests. This method consists of coating CNT tips with a $1 \mu \mathrm{m}$-thick layer of aluminum, which results in delamination during the onedge indentations. Delamination does not occur during the on-edge indentations in the as-synthesized forests. This emphasizes the significant effect that mechanical constraints can have on the occurrence of delamination and informs the design of structures to take advantage of this phenomenon.

Macro-compression testing was performed on CNT forests grown on $1 \mathrm{~cm} \times 1 \mathrm{~cm} \mathrm{Si} \mathrm{substrates.} \mathrm{Nominal} \mathrm{pressures}$ of 50 and $100 \mathrm{kPa}$ were applied to the CNT forests by placing $0.5 \mathrm{~kg}$ and $1 \mathrm{~kg}$ weights on the samples. SEM images taken from deformed CNT forests are shown in Fig. 1. Periodic buckles form close to the substrate (Fig. 1(a)), as was the case in the indentation of CNT forests grown with the same recipe. ${ }^{4}$ The location and commencement of such folds has been shown to be a function of the local density, tortuosity, and entanglement of the CNTs, which commonly vary along the height of CNT forests. ${ }^{1,4,5}$ Buckle formation is not uniform in any of the compressed macroscale CNT forests. The number of buckles along any given edge region range from zero to $\sim 20$. This is likely due to the inherent nonuniformity in the height of the CNT forests - the height variance in each sample was approximately 10\%-50\%, which led to a nonuniform distribution of the compressive stresses within the sample. Height variation is a common consequence of growing the macroscale CNT forests by chemical vapor deposition $(C V D)^{19,34}$ likely caused by flow and temperature variations in the growth chamber. We observe a local delamination of the CNT forest films from 
(a)

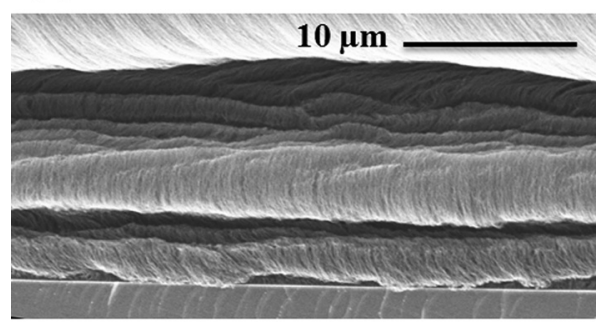

(b)

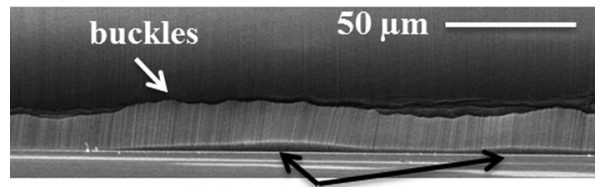

delamination area

(c)



FIG. 1. SEM images of the CNT forests base, illustrating permanent deformation after macro-compression. (a) SEM image of an edge region with multiple buckles but no delamination. (b) SEM image of a location illustrating multiple buckles and delamination of CNTs. (c) A magnified view of a region where CNTs buckled and interface delamination occurred.

the growth substrates (Figs. 1(b) and 1(c)), which occurs in all tested CNT forests within a span of $1 \mathrm{~mm}$ of the edge of the sample under $50 \mathrm{kPa}$ of nominal pressure. The rest of the edge-a span of $9 \mathrm{~mm}$-remains bonded. The lateral extent of the delaminated region increases to greater than $2 \mathrm{~mm}$ when the nominal pressure is doubled. No such delamination is observed in micro-scale flat punch indentation testing of CNT forests grown with the same recipe and loaded to indentation stresses higher than the nominal stress applied in macroscale compression. ${ }^{4}$

Similar delamination occurs in micro-indentation of CNT forests whose top surface is coated with a $1 \mu \mathrm{m}$-thick $\mathrm{Al}$ layer, deposited through e-beam evaporation. Such delamination is not observed during identical tests on the uncoated samples. Indentations to the depth of $20 \mu \mathrm{m}$ were performed with an $80 \mu \mathrm{m}$ wide flat punch on the edge of the $140 \mu \mathrm{m}$ tall CNT forest coated with a $1 \mu \mathrm{m}$-thick Al. Indentations were performed in situ using a dedicated nanomechanical instrument, SEMentor, ${ }^{4,5}$ a combination of SEM and a nanomechanical module similar to a nanoindenter. SEMentor allowed for direct observation of the deformation while concurrently gathering load and displacement data. Fig. 2 illustrates the deformation mechanism of such a representative CNT forest coated with a $1 \mu \mathrm{m}$-thick Al film. Images are frames from the recorded video in SEMentor (see supplementary material ${ }^{35}$ ). Fig. 2(a) shows the edge of the coated CNT forest upon its initial contact with the indenter before the application of pressure. At very small strains, a buckle initiates at $35 \mu \mathrm{m}$ above the substrate, which represents $25 \%$ of the total height (Fig. 2(b)). Lateral propagation of this buckle is the dominant deformation mode up to a strain of 5.5\%, when the CNT film begins to detach from the
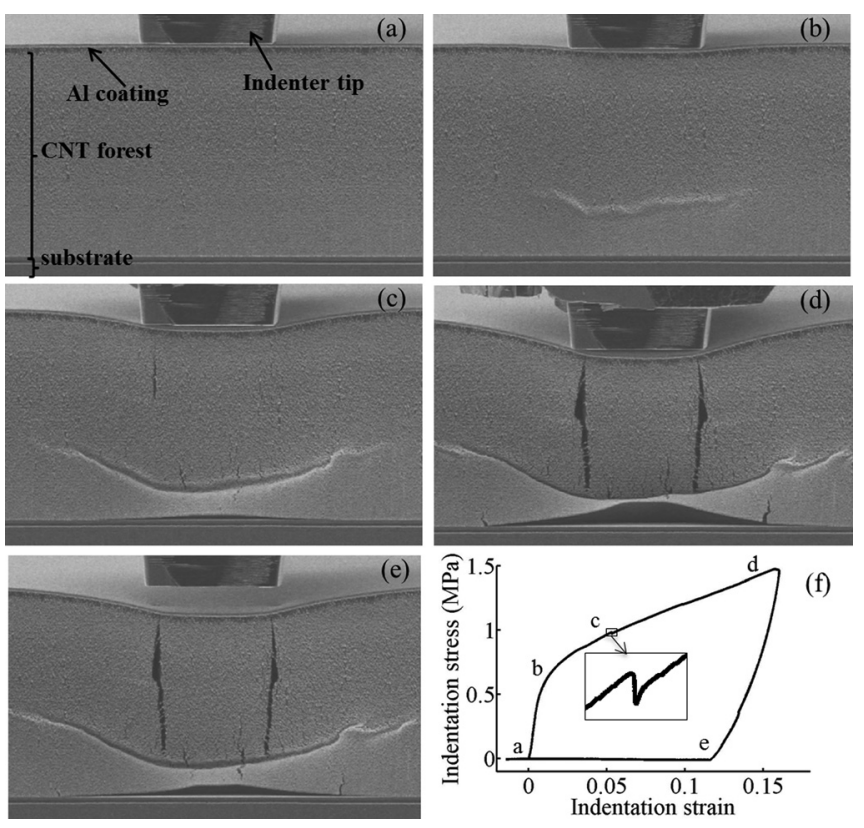

FIG. 2. SEM micrographs (a-e) and stress-strain data (f) illustrating the deformation of a CNT forest coated with a $1 \mu \mathrm{m} \mathrm{Al} \mathrm{film} \mathrm{that} \mathrm{was} \mathrm{indented} \mathrm{at}$ its edge. Images correspond to points a-e on the indentation stress-strain curve in (f).

substrate directly underneath the indenter and vertical cracks appear in the film along the projected indenter edges (Fig. 2(c)). Fig. 2(d) illustrates the maximum displacement of the indenter. At this point, the detached region expands laterally by $230 \mu \mathrm{m}$, and the vertical distance between the delaminated CNT forest base and the substrate reaches a maximum of $17 \mu \mathrm{m}$. Vertical cracks also propagate along the CNT heights and span over $70 \%$ of the film thickness but do not appear to break through the top Al coating. The CNT tips remain adherent to the coating throughout the test. Approximately, $30 \%$ of the total deformation is recovered upon unloading (see Figs. 2(e) and 2(f)). The recovery for the crack opening along the delaminated CNT-substrate interface on the edge is equivalent to the difference between the maximum and the final vertical dimension of the opening, divided by the maximum vertical dimension. This recovery is calculated to be approximately $50 \%$ (Figs. 2(d) and 2(e)).

The indentation stress-strain data are shown in Fig. 2(f). The axial indentation strain is calculated by dividing the measured vertical displacement by the initial height of the CNT forest, averaged over $1 \mathrm{~cm}$ width. To calculate the axial indentation stress, the nano-indenter force is divided by the homogenized contact area underneath the indenter. This area is equivalent to the nanoindenter footprint and remains constant during the test. Data points corresponding to the images in Figs. 2(a)-2(e) are marked on the indentation stress-strain data in Fig. 2(f) and reveal the presence of two distinct deformation regimes: (1) before buckling, characterized by a linear loading slope of $75 \mathrm{MPa}$, and (2) after buckling, where the stress vs. strain relationship remains linear but the slope decreases to 4-6 MPa. Transition between the two regimes occurs gradually, over a strain range of $1 \%-2 \%$. Indentation stress dropped from 885 to $870 \mathrm{kPa}$ at point (c) (see inset in Figure 2(f)). This drop is likely due to the initiation of 
delamination and formation of vertical cracks, which occur faster than the prescribed displacement rate. At this point, the load drops to maintain the constant prescribed displacement rate and the loading slope decreases from 6 to $4.7 \mathrm{MPa}$ (point (c)). In situ SEM video of the indentation and the corresponding video of the stress-strain curve are provided in the supplementary material. ${ }^{35}$ Such a deformation signature and concurrent stress-strain data are consistent over multiple locations (Figure S2 in the supplementary material ${ }^{35}$ ).

To gain insight into the stress distribution within the CNT forest that causes its delamination from the substrate, we employed the Euler-Bernoulli beam bending theory. This theory provides a means for stress calculation under a small deflection in an elastic, isotropic, and homogenous beam. Although the classic beam bending theory does not fully capture the complexities within the CNT forest structure such as inelasticity, inhomogeneity, and anisotropy, it provides useful information about the likely stress distribution under load. We show that it provides a reasonable estimation of the tensile stresses when combined with the deformations observed in situ and the ex situ measurement of the local effective elastic modulus. The classic beam bending theory dictates that the bending moment, $M$, and curvature, $\kappa$, are related by

$$
M=E I \kappa=\frac{E I}{R},
$$

where $E, I$, and $R$ represent the elastic modulus, second moment of inertia, and radius of curvature, respectively. The strong van der Waals driven interactions among the CNTs render Eq. (1) ineffective in describing the bending of each individual nanotube. Instead, it is reasonable to apply Eq. (1) to describe the deformation of coalesced groups of CNTs that form because of van der Waals interactions and therefore buckle in unison. Although the eventual buckling of such bundles of CNTs is inelastic, their initial bending before buckling is observed to be mainly elastic. This is evidenced by the greater than $50 \%$ recovery in the crack opening displacement along the delaminated CNT-substrate interface on the edge upon release of the load (Figs. 2(d) and 2(e)). To account for the inhomogeneity of the CNT forests along the height, the effective moduli of the base of CNT forests were measured by ex situ nanoindentation into the base of a macroscale CNT forest. The CNT forest was separated from the growth substrate by tensile loading and the nanoindentation was performed using a conical tip (more details are provided in the supplementary material ${ }^{35}$ ).
A side view of the CNT deformation in the image shown in Fig. 3(a) is depicted schematically in Fig. 3(b), which illustrates the out-of-plane bending of the structure in the $y$-direction. The curvature observed in the deformed CNT forest provides evidence of the presence of a bending moment in the direction schematically depicted in Fig. 3(c). This bending moment produces the normal tensile and compressive stresses. A differential element of the curved section (marked in Fig. 3(b) with a circle) is used in Fig. 3(c) to demonstrate the bending moment and the stress distribution projected in two dimensions. The bending moment in the depicted direction induces tensile stresses on the outer surface of the element. The normal stress due to bending, $\sigma$, is related to the bending moment, $M$, by

$$
\sigma=\frac{M y}{I},
$$

where $y$, depicted in Fig. 3(c), represents the distance from the neutral axes. By combining Eqs. (1) and (2), the tensile stress at the CNT-substrate interface at the beginning of delamination is estimated by

$$
\sigma=\frac{E y_{\max }}{R},
$$

where $y_{\max }$ and $R$ are estimated using the deformations observed in the in situ SEM videos and $E$ is estimated from the ex situ nanoindentation testing of the base of CNT forests released from the substrate (more details are provided in the supplementary material ${ }^{35}$ ). Substituting the estimated values into Eq. (3) results in $\sigma$ in the range of $0.4-0.8 \mathrm{MPa}$ at incipient delamination.

To compare this analytical estimate with experimentally measured interfacial strength, we perform macroscale tensile testing of the substrate-supported CNT forests. The tensile experiments, which are performed by applying tensile loads on grips bonded to both the growth substrate and CNT tips, resulted in full delamination of CNT forests from the substrates and reveal the adhesion strength between CNT forest and substrate to be between 0.2 and $0.4 \mathrm{MPa}$. This range of adhesive strength falls within the same order of magnitude as the theoretically estimated tensile stresses due to bending proposed above, which lead to delamination at the interface. This agreement suggests that the tensile stresses developed in the entangled bundles of CNTs after buckling are sufficiently high to drive the delamination between the CNT forest and the growth substrate. This strengthens the evidence

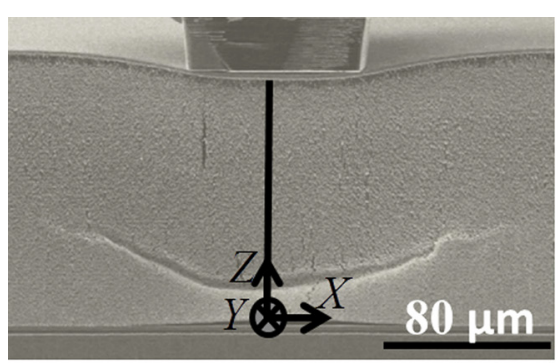

(a)


(b) (c)

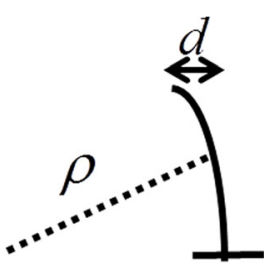

(d)
FIG. 3. (a) Micrograph from Fig. 2(c) with the addition of a vertical line at the middle of the indenter to guide the eye. The inward arrow ( $x$ inside a circle) is the direction of the buckling, (b) illustration of the side view of the out-of-plane deformation of the CNT forest along the vertical line in (a). The left arrow shows the direction of the buckling. (c) Schematic of the bending moment and 2dimensional stress distribution in an element highlighted in (b). (d) Curvature of the section of CNT forest under the buckle location. 

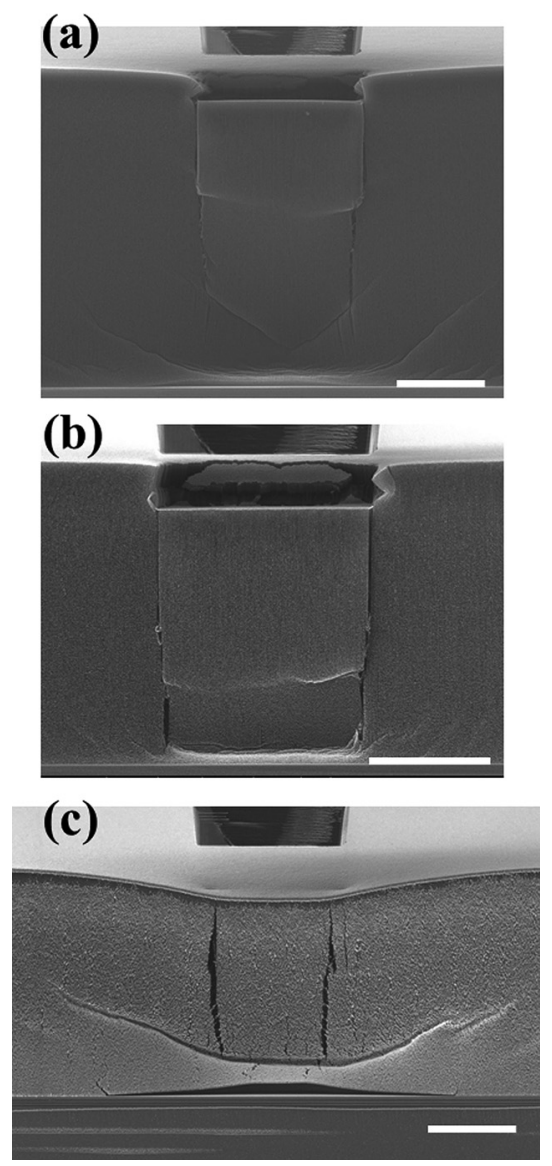

FIG. 4. Post-indentation deformation morphologies on the edge of (a) an uncoated CNT forest and CNT forests coated with (b) $100 \mathrm{~nm}$ and (c) $1 \mu \mathrm{m}$ Al films. Scale bar in each image corresponds to $50 \mu \mathrm{m}$.

that tensile loading due to out-of-plane bending of the CNT forest is the main reason for delamination.

No buckling-followed-by-delamination behavior, as observed in the Al-coated CNT samples, is observed in the uncoated CNT forests when comparing samples indented to the same depth, $30 \mu \mathrm{m}$ (Ref. 4) (Fig. 4(a)). This suggests that even if the tensile stresses are also developed within the uncoated CNT forests, they are lower than the interfacial adhesion. The as-grown samples had more relaxed mechanical boundary constraints, which enabled additional mechanisms to carry the strain, for example, by forming vertical shear offsets along the indenter edges that propagated to the surface (Fig. 4(a)). This causes the CNTs directly under the indenter to collectively separate from the rest of the CNT forest as a block, presumably because of their low resistance to shear as has been previously reported. ${ }^{4,8,9}$ In contrast, the rigid constraint at the top of the coated CNT forests drives their deformation via out-of-plane bending of the buckled area, similar to Mode I crack opening. Larger crack-opening displacements correspond to lower radii of curvature, which in turn generate a greater bending moment and higher tensile stress acting on the interface between the CNT forest and the substrate. On-edge indentation of similar CNT forests coated with $10 \times$ thinner $\mathrm{Al}$ films of $100 \mathrm{~nm}$ results in fracture of the coating and in deformation mechanisms similar to those in the uncoated CNT forests, i.e., with no delamination (Fig. 4(b)). SEM images of the three characteristic deformation morphologies, corresponding to no coating, $100 \mathrm{~nm}$ - thick $\mathrm{Al}$ coating, and $1 \mu \mathrm{m}$-thick $\mathrm{Al}$ coating are illustrated in Fig. 4.

Different from the delamination of the coated CNT samples as a result of the top strain constraint in microindentation, the detachment of the localized interfacial regions (Fig. 1) is likely caused by the non-uniform heights of the CNTs in the macro-compressed samples. The variation in height was measured to be on the order of tens of microns over the lateral extent of hundreds of microns (Fig. S3 in the supplementary material ${ }^{35}$ ). Compressing such a structure results in the originally taller CNTs bending and buckling first, causing lateral displacements within the forest before full contact with all CNTs is established. This likely leads to the formation of local stress concentrations within the samples, i.e., generating the local tensile stress. This tensile stress drives the detachment of certain interfacial regions. The non-constant height distribution likely has a minimal effect during the microscale tests of CNT forests because the wavelength of their height variation is on the order of the nanoindenter dimensions or larger.

In summary, we describe the mechanisms of delamination of 130-190 $\mu$ m-thick CNT forest films, grown via CVD, from their $\mathrm{Si}$ substrates. Both macro-compression experiments of as-grown CNT forests and micro-compression experiments of $\mathrm{Al}$ coated CNT forests display loss of interfacial cohesion at sufficient deformation. In macroscale compression of as-grown CNT forests, localized delamination initiates under nominal pressure as low as $50 \mathrm{kPa}$. The mechanism for delamination under macro-compression may be explained by the development of local stress concentrations within the film, which imposes a local tensile load that is sufficiently high to overcome the adhesion strength between CNTs and the substrate. These local stress inhomogeneities are likely a result of the height variation at the CNT forest surface and the van der Waals interactions between CNTs. In the case of micro-scale indentation of the CNT forest coated with a $1 \mu \mathrm{m}$-thick $\mathrm{Al}$ film, the rigid constraint formed as a result of the applied metal coating is responsible for the delamination. Simple analytical beam bending theory estimates the magnitude of the tensile stress to be on the same order of magnitude as the measured CNT-substrate adhesion strength, $0.4-0.8 \mathrm{MPa}$. These findings demonstrate that tuning the mechanical boundary constraints provides an important design parameter for attaining greater reliability in devices and materials that utilize CNT forests. For example, continuous or percolative thermal and electrical conduction paths through the CNTs can be severed by delamination, which would cause decay in the functionality of the CNT forest film as an interface material. ${ }^{36}$ Such a tunable delamination can also be used in the design of micro-electromechanical systems. For instance, decreased electrical conductance in CNT forests coated with metal films could potentially be utilized to design nano-based mechanical pressure switches and impact sensors that work based on interface delamination.

The details of the experimental procedures are as follows. CNT forests were grown on $1 \mathrm{~cm} \times 1 \mathrm{~cm}$ Si substrates coated with $\mathrm{Ti}(30 \mathrm{~nm}) / \mathrm{Al}(10 \mathrm{~nm}) / \mathrm{Fe}(3 \mathrm{~nm})$ by electron beam evaporation (e-beam). Low pressure CVD (LPCVD) was used for synthesis of CNT forests in a commercial CVD system (Black Magic Pro 4", Aixtron SE). The growth 
temperature was $\sim 750^{\circ} \mathrm{C}$ and acetylene was used as the carbon source gas. The chamber pressure was approximately 10 mbar using a $\mathrm{C}_{2} \mathrm{H}_{2} / \mathrm{H}_{2}$ mixture. A growth time of $5 \mathrm{~min}$ was used and the CNT forest height ranged from 130-190 $\mu \mathrm{m}$. The recipe produced multiwall CNTs with diameter ranges determined by transmission electron microscope (TEM) to be $7.2 \pm 1.4 \mathrm{~nm}$. The average number of walls was measured to be 6 . TEM images are provided in previous work. ${ }^{4}$ Aluminum was deposited on top of some of the CNT forests by e-beam evaporation. A deposition rate of 1 $\AA$ A/s was used.

Nanoindentation experiments were performed in a custom-built in situ nanomechanical instrument, SEMentor, comprised of a nanoindentation arm (Nanomechanics, Inc.) inside a field emission scanning electron microscope (SEM) (FEI Quanta200 FEG). A rectangular cross section $(60 \mu \mathrm{m} \times 80 \mu \mathrm{m})$ diamond flat punch indenter tip was used. Nanoindentation experiments were performed by loading to a maximum displacement of $20-30 \mu \mathrm{m}$ followed by complete unloading. A displacement rate of $50 \mathrm{~nm} / \mathrm{s}$ was used for loading and unloading of the CNTs. The average loading modulus for each stage of loading was measured by dividing the increase in stress by the increase in strain. Additional details on the SEMentor and steps ensuring measurement accuracy are presented in prior work. ${ }^{4,5}$

Macroscale compression and tension tests were performed on CNT forests grown on $1 \mathrm{~cm} \times 1 \mathrm{~cm}$ substrates. Compression testing was performed by placing weights on top of the CNT forest. The weights were centered as much as possible to create the most possible uniform loading. The compressed samples were then imaged in SEM to observe the deformations.

For tension testing, the growth substrate and CNT forest top surface were bonded to loading grips using superglue and carbon tape respectively. Then, vertical displacement with the rate of $100 \mu \mathrm{m} / \mathrm{s}$ were applied in a DTS compression/tension loading unit up to the point of delamination of CNTs from substrate. To prevent failure due to bending moments, a flexible string was used to connect the top grip to the instrument (Fig. S1 in the supplementary material ${ }^{35}$ ). The load at which delamination occurred (the maximum load in the load-displacement curve) was divided to the sample area to calculate the tensile strength. The released bases of the CNT forests were then tested via ex situ nanoindentation to obtain the collective modulus of the base of CNT forests (more details of the ex situ nanoindentation testing in the supplementary material ${ }^{35}$ ).

This work was partially supported by DARPA and the Space and Naval Warfare (SPAWAR) Systems Center, Pacific under Contract No. N66001-09-C-2013. J.R.G. and S.B.H. gratefully acknowledge the financial support from the Institute for Collaborative Biotechnologies (ICB) through grant W911NF-09-0001 from the U.S. Army Research Office. The content of the information does not necessarily reflect the position or the policy of the Government, and no official endorsement should be inferred.

${ }^{1}$ A. Y. Cao, P. L. Dickrell, W. G. Sawyer, M. N. Ghasemi-Nejhad, and P. M. Ajayan, Science 310(5752), 1307 (2005).
${ }^{2}$ M. Bedewy, E. R. Meshot, H. C. Guo, E. A. Verploegen, W. Lu, and A. J. Hart, J. Phys. Chem. C 113(48), 20576 (2009).

${ }^{3}$ E. R. Meshot, M. Bedewy, K. M. Lyons, A. R. Woll, K. Anne Juggernauth, S. Tawfick, and A. John Hart, Nanoscale 2(6), 896 (2010).

${ }^{4}$ P. P. S. S. Abadi, S. B. Hutchens, J. R. Greer, B. A. Cola, and S. Graham, Nanoscale 4(11), 3373 (2012).

${ }^{5}$ S. B. Hutchens, L. J. Hall, and J. R. Greer, Adv. Funct. Mater. 20(14), 2338 (2010)

${ }^{6}$ Y. Gao, T. Kodama, Y. Won, S. Dogbe, L. Pan, and K. E. Goodson, Carbon 50(10), 3789 (2012).

${ }^{7}$ S. Pathak, E. J. Lim, P. P. S. S. Abadi, S. Graham, B. A. Cola, and J. R. Greer, ACS Nano 6(3), 2189 (2012).

${ }^{8}$ S. B. Hutchens, E. Decolvenaere, S. Pathak, and J. R. Greer, Mater. Today 15(3), 127 (2012).

${ }^{9}$ S. Pathak, N. Mohan, P. P. S. S. Abadi, S Graham, B. A. Cola, and J. R. Greer, J. Mater. Res. 28(7), 984 (2013).

${ }^{10}$ M. R. Maschmann, G. J. Ehlert, S. J. Park, D. Mollenhauer, B. Maruyama, A. John Hart, and J. W. Baur, Adv. Funct. Mater. 22(22), 4625 (2012).

${ }^{11}$ A. A. Zbib, S. D. Mesarovic, E. T. Lilleodden, D. McClain, J. Jiao, and D. F. Bahr, Nanotechnology 19(17), 175704 (2008).

${ }^{12}$ A. Qiu, D. F. Bahr, A. A. Zbib, A. Bellou, S. D. Mesarovic, D. McClain, W. Hudson, J. Jiao, D. Kiener, and M. J. Cordill, Carbon 49(4), 1430 (2011).

${ }^{13}$ H. Malik, K. J. Stephenson, D. F. Bahr, and D. P. Field, J. Mater. Sci. 46(9), 3119 (2011).

${ }^{14} \mathrm{~A}$. H. Slocum and O. Yaglioglu, Carbon nanotube based electromechanical probes (Massachusetts Institute of Technology, 2007).

${ }^{15}$ C. M. McCarter, R. F. Richards, S. D. Mesarovic, C. D. Richards, D. F. Bahr, D. McClain, and J. Jiao, J. Mater. Sci. 41(23), 7872 (2006).

${ }^{16}$ J. R. Raney, A. Misra, and C. Daraio, Carbon 49, 3631 (2011).

${ }^{17}$ M. R. Maschmann, Q. Zhang, R. Wheeler, F. Du, L. Dai, and J. Baur, ACS Appl. Mater. Interfaces 3, 648 (2011).

${ }^{18}$ H. J. Qi, K. B. K. Teo, K. K. S. Lau, M. C. Boyce, W. I. Milne, J. Robertson, and K. K. Gleason, J. Mech. Phys. Solids 51(11-12), 2213 (2003).

${ }^{19}$ M. R. Maschmann, Q. H. Zhang, F. Du, L. M. Dai, and J. Baur, Carbon 49(2), 386 (2011).

${ }^{20}$ T. Tong, Y. Zhao, L. Delzeit, A. Kashani, M. Meyyappan, and A. Majumdar, Nano Lett. 8(2), 511 (2008).

${ }^{21}$ S. D. Mesarovic, C. M. McCarter, D. F. Bahr, H. Radhakrishnan, R. F. Richards, C. D. Richards, D. McClain, and J. Jiao, Scr. Mater. 56(2), 157 (2007).

${ }^{22}$ H. Radhakrishnan, S. Mesarovi, C. M. Mccarter, D. F. Bahr, and C. D. Richards, Metalurgija 13(4), 301 (2007).

${ }^{23}$ C. Cao, A. Reiner, C. Chung, S. H. Chang, I. Kao, R. V. Kukta, and C. S. Korach, Carbon 49, 3190 (2011).

${ }^{24}$ A. Qiu, S. P. Fowler, J. Jiao, D. Kiener, and D. F. Bahr, Nanotechnology 22, 295702 (2011).

${ }^{25}$ J. Suhr, P. Victor, L. Ci, S. Sreekala, X. Zhang, O. Nalamasu, and P. M. Ajayan, Nat. Nanotechnol. 2(7), 417 (2007).

${ }^{26}$ S. Pathak, Z. G. Cambaz, S. R. Kalidindi, J. G. Swadener, and Y. Gogotsi, Carbon 47(8), 1969 (2009).

${ }^{27}$ R. L. D. Whitby, S. V. Mikhalovsky, and V. M. Gun'ko, Carbon 48(1), 145 (2010).

${ }^{28}$ Q. Zhang, Y. C. Lu, F. Du, L. Dai, J. Baur, and D. C. Foster, J. Phys. D: Appl. Phys. 43, 315401 (2010).

${ }^{29}$ J. Wang and M. Gutierrez, Micro Nano Lett., IET 2(4), 111 (2007).

${ }^{30}$ C. Daraio, V. F. Nesterenko, S. Jin, W. Wang, and A. M. Rao, J. Appl. Phys. 100, 064309 (2006).

${ }^{31}$ Y. Lu, J. Joseph, Q. Zhang, M. Maschmann, L. Dai, and J. Baur, Experimental Mechanics 52(9), 1551-1554 (2012).

${ }^{32}$ J. W. Hutchinson, M. D. Thouless, and E. G. Liniger, Acta Metall. Mater. 40(2), 295 (1992).

${ }^{33}$ M. D. Thouless, J. W. Hutchinson, and E. G. Liniger, Acta Metall. Mater 40(10), 2639 (1992).

${ }^{34}$ X. J. Wang, J. D. Flicker, B. J. Lee, W. J. Ready, and Z. M. Zhang, Nanotechnology 20(21), 215704 (2009).

${ }^{35}$ See supplementary material at http://dx.doi.org/10.1063/1.4802080 for the in situ SEM video of flat-punch nanoindentation of the CNT forest coated with $1 \mu \mathrm{m} \mathrm{Al}$, video of the stress-strain curve, repeat of the microindentation of the edge of the Al-coated CNT forest, details on the estimation of tensile strength, micro-indentation testing at the middle of the Al-coated CNT forest, and SEM images showing the effects of nonuniformity of CNT forest height.

${ }^{36}$ B. A. Cola, X. Xu, T. S. Fisher, M. A. Capano, and P. B. Amama, Nanoscale Microscale Thermophys. Eng. 12(3), 228 (2008). 